A CALCULUS OF UNUSUAL SIZE REMOVED FROM THE URETER BY OPERATION.

BY FRANK KIDD, F.R.C.S. ENG.,

SURGKON TO, AND SURGEON IN CEARGE OF THF GENTO-THAMTY DEPARTMENT, LONDON HOSPITAL.

I HAVE never seen a stone as large as the one here described removed from the pelvic portion of the ureter, and I should think it must be a record. A stone of such dimensions could hardly have developed in a normal ureter. The ureter was dilated because of the congenital stricture of its mouth, which explains how it became possible for so large a stone to grow therein. Apparently the kidney and ureter had become infected with a mild strain of staphylococcus, which had decomposed the urine and caused a deposit of phosphates which had developed into a stone; in other words, the stone was probably secondary not only to the stricture, but also to an infection. consisted of red blood corpuscles, pns cells, acid phosphate crystals, and the Strphylococcrs albus. The $X$ ray exaruination showed a large oval shadow in the region of the upper portion of the right pelvic ureter, as shown in the appended photograph.

On Sept. 4th Tadministered spinal anæsthesia. With the cystoscope I could see prolapse of the mouth of the right ureter, with cyst formation and intermittent ballooning of the cyst as the urine came down from the kidney. This, although a rare condition, is well recognised by urologists, and has been named "uretero. cele." The primary condition is a congenital stricture of the mouth of the ureter, with secondary dilatation of the ureter and prolapse of the dilated ureter into the bladder. By rectal examination the dilated ureter could be felt, but the stone was too high up in the pelvis to be felt.

Operation.--I cut down in the right groin through a muscle-splitting incision $3 \frac{1}{4}$ in. long, as described by me in THE LANCET of June 7th. 1913. The peritoneum was pushed aside and the ureter identified at the pelvic brim as a thin-walled dilated tube the size of a coil of small intestine. The stone lay below the pelvic brim. fixed to the sicle of the pel vis by peri-ureteric fibrosis.

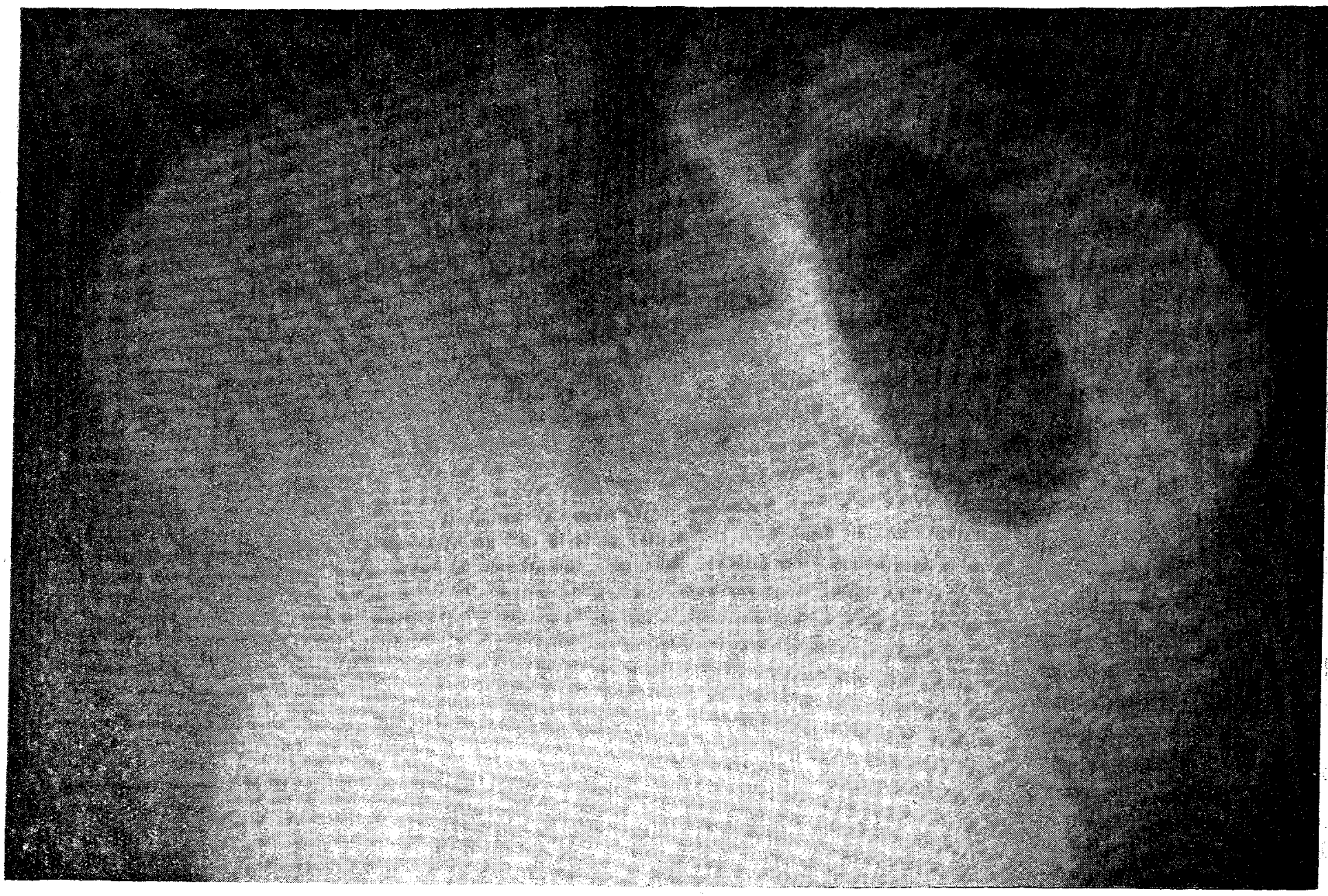

$\mathrm{X}$ ray photograph showing actual size of calculus.

The patient, a young man of 18 years, was admitted to my wards at the London Hospital on August 31st, 1919. He had previously been seen by Mr. A. J. Walton, who very kindly transferred him to my care. In 1907 he had had a stone in the bladder, which had been crushed and removed. In 1908 he had had slight pains in the right loin. After that date he had had no more trouble until eight weeks before admission. At that time a dull, aching pain developed in the right iliac fossa, which lasted four days. A few weeks later the pain recurred and became more acute and was sufficiently bad to lead him to come up for advice and relief. He had never had increased frequency of micturition or any other symptoms.

On examination the urine was acid, contained no albumin, but a moderate haze of thin pus. The deposit
The ureter was opened well above the stone by a longitudinal incision $1 \frac{1}{2} \mathrm{in}$. long, and pus and urine gushed out. The stone lay fixed in a large pouch; the upper end of the pouch was cut open and the stone was easily removed. Bougies could then be passed into the bladder. The wall of the ureter was re-stitched with tine catgut in two layers, a small tube was placed at the site of the stitching and brought out through the abdominal wound; the abdominal wound was stitched up with catgut in layers. The operation took 30 minutes.

Course.-A few drops of urine leaked from the tube for 24 hours; after that there was no more leakage, and the tube was removed. On the fourth day the stitches were removed; on the eighth day the wound had healed by first intention. except for the tuhe hole. The patient 
left the hospital on the sixteenth day, soundly healed and with no further symptoms. The stone measured $27 \mathrm{in}$. in its long diameter and $1 \mathrm{in}$. in its short diameter, and weighed $1 \mathrm{oz}$. It consisted of phosphates, with a trace of oxalates, and contained no cystin, no carbonate, and no uric acid.

\section{Comments on the Case.}

It is interesting to note how very few symptoms had been caused by the stone. As a general rule, the larger the stone the fewer the symptoms. Since I wrote my paper on the removal of ureteric stones I have seldom found it necessary to operate for stones in the lower portion of the ureter. Most ureteric stones in the pelvic portion of the ureter lie just above the bladder, and are prevented from entering the bladder by the narrowness of the opening of the lower end of the ureter. I find by experience that I can slit up the mouth of the ureter with a pair of operating scissors, through the operating cystoscope, lubricating the stone with parolene, and that a few days or weeks later these stones are passed naturally without any further trouble. In this case the stone was so large that it was clearly necessary to operate by open incision.

B. S. Barringer ${ }^{1}$ describes a case of double ureterocele with a small stone lying in one of them. He cut off the top of the ureteroceles with the operating cystoscope, and the stone was then passed naturally. I have previously seen two cases of ureterocele; in one I treated the condition by open operation, and in the other I cut off the top of the projection through the operating cystoscope; on both occasions results were satisfactory. I hope to operate at a later date on the ureterocele present in the case reported here.

\section{DIVERTICUIITIS.}

'Being some Remarks made in the Course of a Discussion at a Meeting of the Section of Surgery (Subsection of

Proctology), Royal Society of Medicine, on Jan. 7 th,

BY G. GREY TURNER, M.S. DURH., F.R.C.S.ENG., HONORARY ASSISTANT SURGEON, ROYAL INFIRMARY, NEWCASTLE-ON-TYNE,

Mr. first introduction to the subject under discussion was in the year 1903, when, as surgical registrar, I saw Professor Rutherford Morison excise what was supposed to be a malignant growth of the sigmoid in a man of 60 . On examining the specimen $I$ found that it was an example of diverticulitis. There were 13 diverticula, with great inflammatory thickening of the bowel wall, but no lesion of the mucous membrane. The man died, and at the post-mortem many diverticula were found from about the middle of the transverse colon to the point at which the sigmoid had been divided, but none in the lower segment of the bowel. The condition was at once recognised, and was correctly described in the hospital notes.

This case is of some interest, because it has been stated by Sir Berkeley Moynihan in his Cavendish lecture for 1912 that he operated in 1906 "upon the first case recognised as chronic diverticulitis of the sigmoid flexure in this country." I always regret that the example to which I have referred was not published at the time, for it would have stimulated an earlier interest in this particular subject. But there was really no excuse for ignorance in the minds of inquiring surgeons, for a well-illustrated abstract of the paper by Ludwig Schreiber appeared in the Medical Review of

1 Transactions of the American Association of Genito-Urinary Surgeons, May, 1914
Reviews about that time, and in THE LANCET for Oct. 24th, 1903, Sir John Bland-Sutton wrote a most suggestive paper "On the Effect of Perforation of the Colon by Small Foreign Bodies, especially in relation to Abscess of an Epiploic Appendage.'

Since then several examples have come under my notice, and they have illustrated all the clinical conditions which have been described.

Description of Cases.

I would like to refer to three cases of particular interest.

The first is that of a female patient, aged 34 years, on whom I operated in 1904 for what was diagnosed as appendicitis with abscess. ${ }^{2}$ I found a hard mass in the ascending colon which so closely simulated a malignant new growth that I thought it best to excise it. On examining the specimen I found the explanation to be an inflammation around a diverticulum in the commencement of the colon. There was also another quiescent saccule on the caput. No other diverticula were noticed in the large bowel during the course of the operation. The patient made a good recovery, and was living and well 15 years later.

The second case must be almost unique. It is that of a man 57 years of age who was admitted to the Newcastle Infirmary in the year 1902 suffering from retention of urine with general peritonitis. As it had not been found possible to relieve the condition by catheter an attempt had been made at his home to puncture the bladder suprapubically, but only liquid faces escaped through the trocar. The man soon died, and at the postmortem I found what I then took to be a malignant growth of the sigmoid, and just above this growth was a large hole leading into the bowel; there was facal extravasation with general septic peritonitis. On looking for an explanation I discovered, free in the rectovesical pouch, a large gall-stone which had evidently ulcerated through the sigmoid just above the growth. An examination of the gall-bladder showed a second calculus projecting from that viscus into the hepatic flexure. The condition was interesting enough, and the specimen was put aside for further investigation, but amid an abounding wealth of pathological material it was neglected until the year 1908, when I was especially interested in the question of gallstone ileus. On re-examination I then found that the condition was a typical example of diverticulitis, and that there was no question of new growth. Unfortunately the specimen has been mislaid, but I am able to show you a drawing which was made at the time.

The third case shows that peritonitis may occur as the result of ulceration of even a small and almost isolated diverticulum, for in this patient only three such diverticula were found after a careful search of the bowel, cut in serial sections.

\section{Atiology, Diagnosis, and Treatment.}

As regards atiology, I have the conviction that the condition will ultimately prove to be congenital in origin. This is in accordance with the general origin of the diverticula with which we are familiar in the various parts of the alimentary canal and in the bladder. In one of my cases, a man who died from peritonitis and who presented diffuse diverticula in the large bowel (42 in number), there were also two large sacculi in the bladder in relation to the left ureter. At the time the latter were attributed to an enlarged prostate, but extended experience of vesical pouches has led me to realise that in many cases these are certainly congenital in origin. We must freely admit that in their early stages all diverticula are probably

1 Since hearing Sir John Bland-Sutton's remarks at the discussion I have taken the opportunity of again carefully reading his paper, div fud it full of diverticulum of the colon, and some of the cases were undoubtedly examples of aivericula not explanation.

explanation. Simulating Appendicitis, The LAXCFT, Sept. 16 the 1905. 